\title{
SURFACE CRACK DETECTION WITH LOW-COST PHOTOACOUSTIC IMAGING SYSTEM
}

\author{
Andreas Setiawan ${ }^{1,2^{*}}$, Gede Bayu Suparta ${ }^{2}$, Mitrayana $^{2}$, Waskito Nugroho \\ ${ }^{1}$ Department of Physics, Faculty of Science and Mathematics, Satya Wacana Christian University, Jl. \\ Diponegoro 52-60, Salatiga, 50711, Indonesia \\ ${ }^{2}$ Department of Physics, Faculty of Mathematic and Natural Science, Universitas Gadjah Mada, Sekip \\ Utara, Yogyakarta, 55281, Indonesia
}

(Received: May 2016 / Revised: February 2017 / Accepted: January 2018)

\begin{abstract}
Photoacoustic measurement in an imaging system is a unique method as it uses optical disturbance but observes its effect acoustically. Acoustical observation increases the quality of a measurement by reducing the scattering effect that often occurs in optical research. However, a few problems, such as cost and complexity, hinder development of a photoacoustic system. This report presents a design of a photoacoustic system using a laser diode and a commercial microphone as the acoustic emission source and sensor, respectively. Analysis of the photoacoustic signal received by the microphone was performed with software-based Fourier transformation, which makes the photoacoustic system simpler and lower in cost. By measuring the amplitude of the signal, the system accurately detects surface micrometer cracks. The report shows that the system is capable of producing a photoacoustic image of an object with microcracks on its surface. The results indicate that the photoacoustic imaging system developed in the experiment is a more promising way to generate images of cracks than optical imaging.
\end{abstract}

Keywords: $\quad$ Cracks; Imaging system; Laser diode; Photoacoustic

\section{INTRODUCTION}

Many researchers have studied fractures in order to develop an improved material inspection technique. A crack occurs due to weakening of a material where its structure is torn because of product defects and environmental effects such as corrosion and fatigue (Gdoutos, 2005). The appearance of cracks on a material indicates that a structure is starting to collapse (Sih \& Chen, 1981; Bouayoune et al., 2017). Transportation research has found that material cracking is one factor promoting mechanical system failure (Craig et al., 2006). To solve these problems, various methods have been developed, such as use of a strain gauge, visual camera, thermography, and many others (Tolev \& Mandelis, 2010; Lantz, 2011; Rupil et al., 2011; Jeong et al., 2012). Those techniques use disturbance and observation of the same type; for example, a visual camera uses optical disturbance as well as optical observation.

Photoacoustic measurement is now being extensively developed to overcome problems associated with the optical imaging system such as diffuse reflection from an irregular surface. It is a distinctive method that uses optical disturbance but observes its effect acoustically (Tam, 1986; Bento et al., 2002; Beard, 2011). Acoustical observation is advantageous as it reduces the scattering effect that often occurs in optical analysis, consequently increasing the quality of the

\footnotetext{
${ }^{*}$ Corresponding author's email: andreas.setiawan@staff.uksw.edu, Tel: +62-298-321212, Fax: +62-298-321433
} Permalink/DOI: https://dx.doi.org/10.14716/ijtech.v9i1.1506 
inspection.

Photoacoustic measurement was first developed in the 1800s, but it was slow and limited due to its complexity (Favro et al., 1980; Wang, 2009). However, the invention of semiconductor and laser technology in the early 1960s has allowed it to become one of the most advanced imaging techniques available today (Ganguly \& Rao, 1981; Luscher et al., 1984; Ni, 2014). Several researchers have reported that applying photoacoustic measurement to detect material cracking yielded satisfactory results (Chigarev et al., 2009; Yan et al., 2012; Mezil et al., 2013; Setiawan et al., 2017). However, they encountered problems due to the high cost and lack of compactness of this technique (Maslov \& Wang, 2008; LeBoulluec et al., 2013; Wang et al., 2014).

Overall, a photoacoustic imaging system consists of three main devices: a beam source, sensor, and amplifier equipped with a data analyzer. Most commonly used beam source is laser-based such as $Q$-switched Nd: YAG; Ti: Saphire, an optical parametric oscillator (OPO) and many others. A laser is used because it has high power and short pulse duration, producing data with a high signal to noise ratio (SNR). Despite its suitability, the photoacoustic signal range is lower than the noise measurement; consequently, direct evaluation of the signal is problematic. A complex method and device for signal recovery, such as a lock-in amplifier, are needed to achieve better results when assessing photoacoustic signals. A lock-in amplifier works by multiplying measured signals from the modulated laser at a low frequency (Vandenbussche et al., 2014). This multiplication process creates a clear distinction between the real assessed signal and noise that appears to be random. Another main part of the photoacoustic system is the acoustic sensor. Ideally, the system should include a sensor with a very wide frequency response to generate a signal with the perfect shape. Typically, photoacoustic signals appear as narrow band pulses. Hence, the sensor should be able to read a frequency range from audible to higher ultrasonic frequencies. The combination of the frequency responses of the sensor and of the amplifier will determine the final result of the detected photoacoustic signal. Limiting the bandwidth of the amplifier will result in the loss of some signal frequencies, which in turn will affect the shape of the signal generated by the sensor. This will complicate data acquisition and analysis. Generally, a laser beam is emitted with a very narrow pulse width in a very short time (microseconds to nanoseconds) to avoid heating the object and achieve high spatial resolution. Thus, the generated acoustic signal will change rapidly depending on the given disturbance. A high-speed recording system is needed to record the details of each change of the signals for good photoacoustic image reconstruction. However, the recording device is complex and expensive, which creates another problem associated with application of a photoacoustic imaging system.

Considering the factors described above, many researchers have attempted to create a more affordable and simpler photoacoustic system. One such innovation uses a laser diode as the beam source (Lopez et al., 2002; Maslov \& Wang, 2008; Wang et al., 2014). It is portable, less expensive, compact, and durable. In addition, the sensors and the data logger are made less complicated and less expensive, but their quality is maintained to achieve better results.

This report describes the innovative procedures of a compact, simple, and low-cost photoacoustic system to detect micro-cracks in materials. The system uses a laser diode and a commercial microphone as the acoustic emission source and sensor, respectively. The laser diode emission source is prepared with a custom-built driver, while the data logger system uses a soundcard integrated into the microphone as the sensor. The software is designed to replace the lock-in amplifier used to record fluctuations in the amplitude of the signal. The procedures and devices used in the experiment show the results of good-quality photoacoustic signals with high SNR. Further assessment is done by conducting an experiment to create a 2D image of a crack on the surface of a metal object. The research and experiments were conducted in the Citra Laboratory 
of Gadjah Mada University from 2016 to 2017. The environmental conditions are explained in Table 1.

Table 1 Environmental parameters

\begin{tabular}{cll}
\hline No. & \multicolumn{1}{c}{ Variable } & \multicolumn{1}{c}{ Value } \\
\hline 1. & Temperature & $31^{\circ} \mathrm{C}$ \\
2. & Pressure & $100 \mathrm{kPa}$ \\
3. & Humidity & $58 \%$ \\
4. & Sample & Aluminum 1060 \\
5. & Sample Purity & AL 99\% \\
6. & Sample Manufacture & KWK Steel Co., Ltd. \\
\hline
\end{tabular}

A more precise image of the crack compared to the common optical imaging method was achieved, although the size of the crack is in the micrometer range. As the design of this system is successful, it is expected to enable the development of an affordable and extensively used photoacoustic system.

This photoacoustic system may be applied in low-visibility environments as it works in acoustic wave domains, where an optical camera cannot be used. In addition, impairment in micro-objects is easier to detect using a laser probe.

\section{EXPERIMENTAL METHODS}

A laser is a monochromatic electromagnetic light with a linearly polarized plane wave, allowing it to deliver energy more efficiently. According to basic theory regarding laser-material interaction, when laser beams fall on the surface of an object, the material is likely to absorb energy (Kruusing, 2008). The optical characteristics of a material affect various physical dynamics in the process (Ristau, 2015). Thus, analysis of objects' optical characteristics will enhance the understanding of laser-material interactions.

Generally, an electric field travelling without absorption through a homogenous medium is given by the following equation:

$$
\vec{E}=\vec{E}_{0} e^{i(2 \pi / \lambda-\omega t)},
$$

where $z$ is the coordinate of the travelling direction of the electric field, $\omega$ is the angular frequency, and $\lambda$ is the wavelength. Based on Drude model, the wavelength can be associated with a refractive index and light velocity using the following equation:

$$
\lambda=\frac{2 \pi}{\omega} \frac{c}{n_{1}} \text {. }
$$

The travelling electric field produces a magnetic field, $H$, which is expressed as follows:

$$
\vec{H}_{0}=n_{1} \varepsilon_{0} c E_{0},
$$

where $\varepsilon_{0}$ is the dielectric constant and $n_{1}$ is the refractive index in a vacuum. The amount of laser energy per unit area can be defined by the following equation:

$$
I=|\vec{E} \times \vec{H}|=n_{1} \varepsilon_{0} \mathrm{c} E_{0}^{2} .
$$

When the lateral distribution of the beams has Gaussian symmetry, the cylindrical coordinates are written as follows:

$$
I(r)=I_{0} \mathrm{e}^{-\mathrm{r}^{2} / \mathrm{w}^{2}}
$$

where $I_{0}$ is the radiation at the center of symmetry $(r=0)$ and $w$ is the radius of the beam. 
Referring to this equation, the total power of the laser is determined as follows:

$$
P=\pi w^{2} I_{0}
$$

This equation will determine the amount of power of the laser interacting with the object exposed to it.

There are three different types of laser-material interactions depending on the characteristics of the materials involved in the interaction (i.e., insulator, semiconductor, or conductor materials). Those materials are different from each other due to the diverse nature of their charge carriers. For conductor materials, optical response is dominated by electron conduction, in which energy gaps are more likely to occur. Electrons containing energy close to the Fermi energy level are commonly called free electrons, and they have a significant impact on the optical characteristics of metallic materials. Free electrons contain no resonance frequency. Therefore, only electronlattice interaction in the collision process contributes to the power of the laser-material interaction. Dielectric function for electrons in a metal is given by the following equation:

$$
\varepsilon=1+\omega_{p}^{2} \frac{-\tau_{e}^{2}+i \tau_{e} / \omega}{1+\omega^{2} \tau_{e}^{2}}, \text { with } \omega_{p}=\sqrt{\frac{N_{e} e^{2}}{m_{e} \varepsilon_{0}}},
$$

where $\omega_{p}$ is plasma frequency. Referring to equation (7), for the range of $\omega<<1 / \tau_{e}$, it is known that $\varepsilon_{1} \approx-\sigma_{0} \tau_{e} / \varepsilon_{0}$ (far infrared region), $\varepsilon_{2} \approx \sigma_{0} / \varepsilon_{0} \omega, n_{1} \approx n_{2} \approx\left(\sigma_{0} / 2 \varepsilon_{0} \omega\right)^{1 / 2}$, and $\alpha=\frac{4 \pi n_{2}}{\lambda}$, $R=\left|\frac{n-1}{n+1}\right|^{2}$. Hence, the reflection and absorption levels of a material can be calculated using the following equations:

$$
1-R=\sqrt{8 \varepsilon_{0} \omega / \sigma_{0}}, \alpha \cong \sqrt{2 \omega \sigma_{0} / \varepsilon_{0} c^{2}}
$$

For an interval time of $1 / \tau_{e}<\omega<\omega_{p}$ (the infrared region for most metals), it is given that $n_{1} \approx \omega_{p} / 2 \omega^{2} \tau_{e} \approx 0$ and $n_{2} \approx \omega_{p} / \omega$, so reflection and absorption function are determined by the following equations:

$$
1-R=\frac{2 \varepsilon_{0} \omega_{p}}{\sigma_{0}}, \alpha \approx \frac{2 \omega_{p}}{c}
$$

Equation 9 describes the process by which a laser comes into contact with a material, the characteristics of which define the mode of interaction. One important parameter in laser-metal interaction is plasma frequency. The laser used in the experiment needs to be set according to the plasma frequency to produce an optimal interaction. A maximal absorption level will be achieved if the frequency of the laser lies in the range of the plasma frequency of the material.
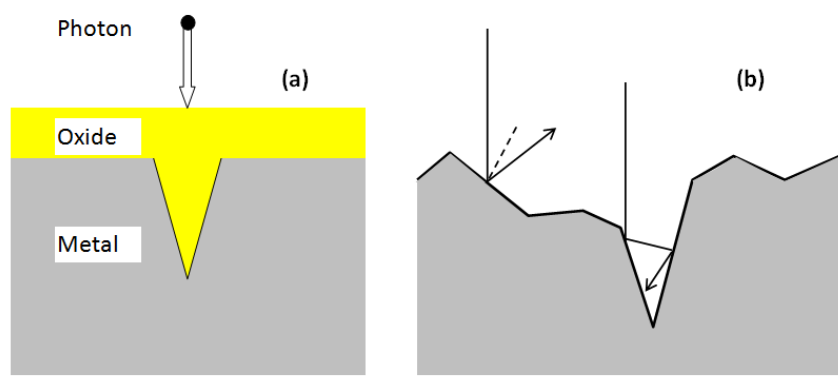

(c)

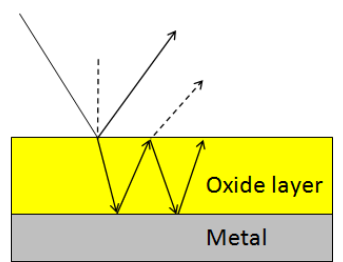

Figure 1 Two mechanisms affecting the absorption level of typical surface crosssections (a). Repetitive reflection is likely to occur where the surface texture causes light to fall at a certain angle (b). An oxide layer traps light (c) (Bergstrom, 2008) 
Since the equations described above focus on electrical interaction, the material's surface is a negligible factor. However, the effects of a material's surface are examined thoroughly in this report. Microscopically, the surface of a material has diverse peaks and valleys. Roughness level is a macro-measurement of variation in a material's texture.

Figure 1 illustrates the sequence of events that occur when a laser interacts with the surface of a material. Geometrically, when beams fall in a certain gap with an incident angle pointing to the material, repetitive reflection of the rays occurs inside the gap (Figure 1b). The repeated interaction between the laser and material will result in a higher level of light absorption, therefore increasing the absorption coefficient. Another condition of laser-material interaction is a light ray trapped in a thin surface layer (Figure 1c). This layer might be produced by pollution or oxides resulting from interaction between the metal and the environment. An oxide layer has a specific refractive index that traps light rays coming from a certain incident angle. Bergstrom (2008) exposed two different lasers, Nd: YLF and Nd: YAG (532 nm and $1064 \mathrm{~nm}$, respectively), to a mild steel object and a stainless steel object to demonstrate the trapping of light in a thin oxide layer. The surface roughness level is measured using a scanning electron microscope (SEM) based on the average size of peaks and valleys, while the absorption and reflection coefficients are measured using a standard reflectometer. The results show that, at the same surface roughness, stainless steel has a higher level of absorption than mild steel. In addition, laser wavelength has no effect on the absorption process; at considerably different wavelengths ( $532 \mathrm{~nm}$ and $1064 \mathrm{~nm}$ ), the absorption curves are similar. This proves that surface roughness is a dominant characteristic affecting absorption in a material along with plasma frequency, which affects the electronic properties of a material. As a result, interaction between laser and material becomes insensitive to the wavelength in roughness surface interaction.

Fluctuations in absorption level can be observed optically by changing image intensity; higher absorption levels will produce a more intense image. The photoacoustic imaging method requires deeper understanding of the association between absorption and acoustic signal as the main factors producing photoacoustic signals. The process of photoacustic imaging begins when the molecules of an object absorb laser photons, followed by radiative and non-radiative relaxation (Montalti et al., 2006). Non-radiative relaxation occurs when the molecules absorb energy and release it through intramolecular redistribution. When this occurs, the object may slightly increase in temperature. The process will continue until equilibrium is achieved, following the equipartition theorem of energy. Radiative relaxation, which involves fluorescence and phosphorescence, may occur if there is a sudden decrease in energy of the molecule as it releases photons. For fluorescence, the transition happens only for molecules with the same energy level (e.g., a change from a singlet to another singlet) and occurs very swiftly (i.e., at a nanosecond scale or less). Phosphorescence occurs when relaxation involves cross-transition between intramolecular systems, such as from a triplet to a singlet. In quantum mechanics, there is only a slight probability that intersystem crossing will take place (Montalti et al., 2006). Therefore, intersystem crossing takes a longer time than radiative relaxation (at a millisecond scale). Collision among molecules is likely to happen during the intramolecular redistribution process. Consequently, excitation of vibrational energy may be converted into translational kinetic energy in molecules. Macroscopically, this energy results in an increase in the temperature of the object. Thus, it is called the photothermal effect. Each collision is very short (i.e., at the picosecond scale) but is a random event that makes the thermal effect last longer (i.e., a thousandth of a microsecond or even a second).

The photothermal effect may be associated with the photoacoustic process. Initially, an object is exposed to laser beams for a short duration. Laser photons will interact with the material and diffuse to a certain depth. An area with a higher absorption level will dominate the photothermal effect during active laser exposure, which causes local expansion to exert a force on the material, 
generating an initial acoustic pressure. Just before the laser stops, the expanded part will contract, producing a mechanical impulse that travels throughout the object to achieve an equilibrium state. This moving dynamic pressure is identified as acoustic waves. Thus, by measuring the acoustic waves generated, the characteristics of an area can be analyzed.

The intensity of the photothermal effect is determined by the amount of energy absorbed by an object. After a laser is exposed to the object (at a picosecond time scale), irradiation in the material will follow a time function, $\phi(x, t)$. However, as the laser pulse period is longer than the equilibrium time, irradiation will only follow the space function, $\phi(x)$. Therefore, total irradiation is given by the following equation:

$$
\Phi(x, t)=\phi(x) f(t) \quad \mathrm{Wm}^{-2}
$$

where $f(t)$ is the temporal laser pulse (without a unit), while the absorbed energy density is $H(x, t)=\mu_{a}(x) \phi(x) f(t)$, with $\mu_{a}$ representing the absorption coefficient $\left(\mathrm{m}^{-1}\right)$. The initial acoustic pressure is defined by the following equation:

$$
p_{0}=\Gamma H,
$$

where $p_{0}$ is the initial acoustic pressure and $\Gamma$ is the Gruneisen parameter (Wang, 2009). This parameter, proposed by Gruneisen in 1912, represents the thermodynamic characteristic of a material and macroscopically defines the ratio of the grating vibration mode, $\omega_{i}$, to the volume, $V$, as follows: $\Gamma_{i}=-\frac{\partial \ln \omega_{i}}{\partial \ln V}$ (Vocadlo et al., 2000).

According to Equation 11, when irradiation and laser duration are kept constant, an increase in photon absorption will be followed by a rise in the amplitude of the acoustic signal. This relation explained the signal amplitude changing process caused by cracks on material surfaces.

Figure $2 \mathrm{a}$ shows the sequence of events that occur when a laser interacts with the rough surface of a gap caused by cracks. The end of a naturally formed crack is rougher than the middle. Thus, when laser beams fall on it at a certain angle, a repetitive reflection will take place, enhancing the energy absorption of the material. Given Equation 11, the amplitude of the acoustic signal will be stronger on the edges of the crack than on the smooth middle part. Thus, cracks can be distinguished from normal regions.

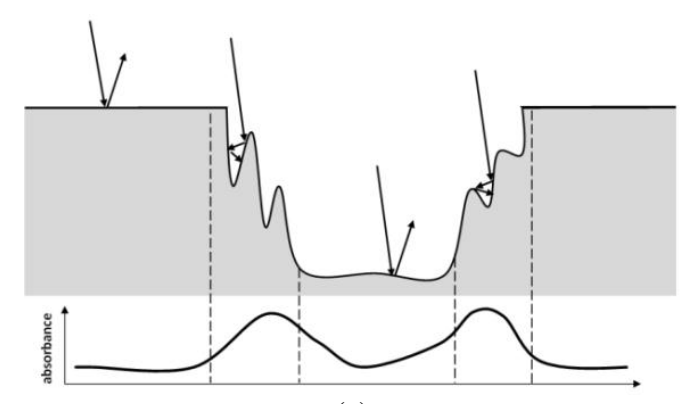

(a)

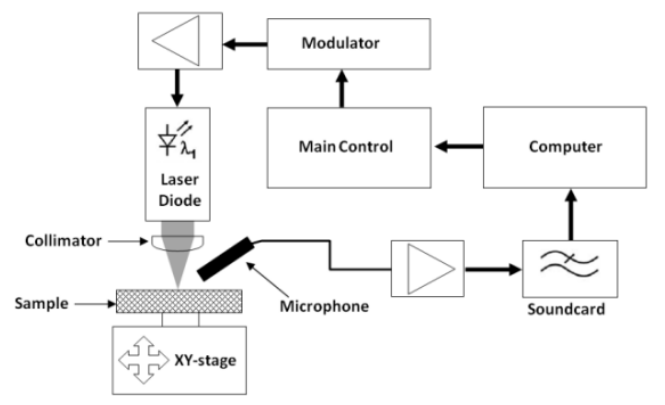

(b)

Figure 2 (a) Cross-sectional fracture. On the edges of the crack, the laser beam is trapped and repetitively reflected. (b) The photoacoustic system scheme for detecting micro-cracks consists of three main parts: a modulator, a laser diode, and a microphone. The microphone, which serves as an acoustic signal detector, is connected to a computer through an integrated amplifier and a soundcard.

Figure $2 b$ shows the experimentation scheme, which consists of three main parts: a unit control, a laser diode, and a microphone. One of the functions of the unit control is to drive the object or sensor for 2D scanning. An $\mathrm{x}-\mathrm{y}$ stage system is positioned on the $\mathrm{x}$ - and $\mathrm{y}$-axes using a bipolar 
motor, SLO-SYN Model M061-FD02E. The motor is powered by a micro-stepping motor stepper driver, which is a very precise movement module. This movement is done by configuring a continuous modulated current-driven bipolar motor. The current is generated by a digital-toanalogue converter (DAC) device. The motor driver will send binary codes to the DAC, which will generate currents in a continuous waveform pattern that serve as input for a transistor amplifier to drive a two-coil motor. The continuous current movement will create a smooth electric field change in both coils, and as a result, the motor blade will move in a relatively precise angular manner. This enables the motor to move at a very small angle to achieve maximum scanning precision. The smallest possible angle is $1.8 \% / 32$, or 6400 steps per rotation. Microstepping allows movement of less than one micrometer, while the micrometer model has $1 \mathrm{~mm}$ linear shift per rotation.

The measurement process begins when the laser diode emits beams focused on the surface of an object that is two-dimensionally moved by the $x-y$ stage. This will generate an acoustic signal that is recorded by the microphone. The result of each measurement is sent to the computer for further processing. The laser used in the experiment is ML520G12 (Mitsubishi), which provides stable oscillation with an emission wavelength of $805 \mathrm{~nm}$ and a maximum power of $500 \mathrm{~mW}$. A Class A transistor amplifier is used to move the laser diode. The amplifier is set to align with the linear laser diode operating current, which ranges from 400 to $690 \mathrm{~mA}$. After this, the amplifier is driven by a modulator with a pulse width of $1 \mu \mathrm{s}$ and repetition of $11 \mathrm{kHz}$. The maximum condition is reached when it is given a current of $690 \mathrm{~mA}$, which is equal to $480 \mathrm{~mW}$ of laser power. A measurement microphone (Behringer ECM8000, 15-20 kHz) integrated with a 16-bit XLR-to-USB sound card with a sampling rate of $48 \mathrm{KHz}$ is used to measure acoustic amplitude. It is an omnidirectional microphone with a maximum sensitivity of $-60 \mathrm{~dB}$. An aluminum plate measuring $5 \times 10 \mathrm{~cm}$ with a $30 \mu \mathrm{m}$ crack in the middle is used as the experimental object. For photoacoustic imaging, the object is mounted on the $x-y$ stage and a $2 \times 2 \mathrm{~mm}$ section is scanned through the middle of the crack. The image is compared by visual imaging using an Examet Union 81837 (Ogawa Seiki Co., Ltd.) digital microscope.

Matlab is the software used in data analysis to identify the signal amplitude from the modulated laser frequency. The purpose of setting a modulated frequency is to simplify the signal separation from a lower noise frequency. Amplitude reading is used to determine the level of pixel intensity to generate a photoacoustic image. Collected data are divided into segments to represent the position of a pixel. The software then creates a frequency spectrum from the segmented data using the fast Fourier transformation (FFT). There are 2,500 segments measuring 50×50 pixels. The total time needed for recording is 25 minutes as each segment is recorded in $1 / 100$ minute (around 0.6 seconds).

According to the manual audacity, WAV 16-bit Pulse Code Modulation (PCM) is selected for data recording. It produces around $5 \mathrm{MB}$ of uncompressed WAV data per minute and a total of $125 \mathrm{MB}$ in 25 minutes. The Audacity recording process uses a project rate of $44,100 \mathrm{~Hz}$. Thus, in 25 minutes there will be $44100 \times 25 \times 60$ samples, or $66,150,000$ samples divided into 2500 segments, each of which consists of 26460 samples. This arrangement is used because FFT requires the number of samples to be a factor of 2 . A sample of $16384\left(2^{14}\right)$ is taken from the 26460 segments. This data segmentation is designed to optimize the number of samples used in the analysis and increase the density of pixels.

\section{RESULTS AND DISCUSSION}

The purpose of this experiment is to detect photoacoustic signals and the effects of modulated laser frequency. Three different frequencies are used in the experiment: $5502 \mathrm{~Hz}, 10194 \mathrm{~Hz}$, and $15013 \mathrm{~Hz}$ (representing low, middle, and high frequency ranges, respectively). A set of recorded 
data is used to generate a frequency spectrum, which is shown in Figure 3. The microphone can respond to a frequency range up to $20 \mathrm{kHz}$ with a decaying amplified response when it reaches a higher range of frequency. The acoustic signal has a sharp peak at the modulated frequency. This shows that the photoacoustic signal generated by the system is highly distinguishable from its environment, promoting a high SNR in further image analysis. Advance measurement of the three different frequency ranges produces a significant peak in the photoacoustic signals, though there is a slight amplitude fluctuation with an average difference of around $10 \mathrm{~dB}$ from the background signals. As shown in Table 2, the signal difference is decreasing as the frequency moves to a lower range. However, this is probably caused by the microphone's response, which tends to be stronger at its lower frequency, enhancing the background signals. Consequently, if the modulated frequency is too low, it will be difficult to detect as it will be overridden by the background noise. In conclusion, the selected modulated frequency did not produce profound results.

Table 2 Photoacoustic signal peaks at low, middle, and high modulated frequencies

\begin{tabular}{ccccc}
\hline No. & Frequency $(\mathrm{Hz})$ & Peak $(\mathrm{dB})$ & Background $(\mathrm{dB})$ & Difference $(\mathrm{dB})$ \\
\hline 1. & 5502 & -43.5 & -54.0 & 10.5 \\
2. & 10194 & -46.9 & -63.0 & 16.1 \\
3. & 15013 & -51.4 & -66.0 & 14.6 \\
\hline
\end{tabular}

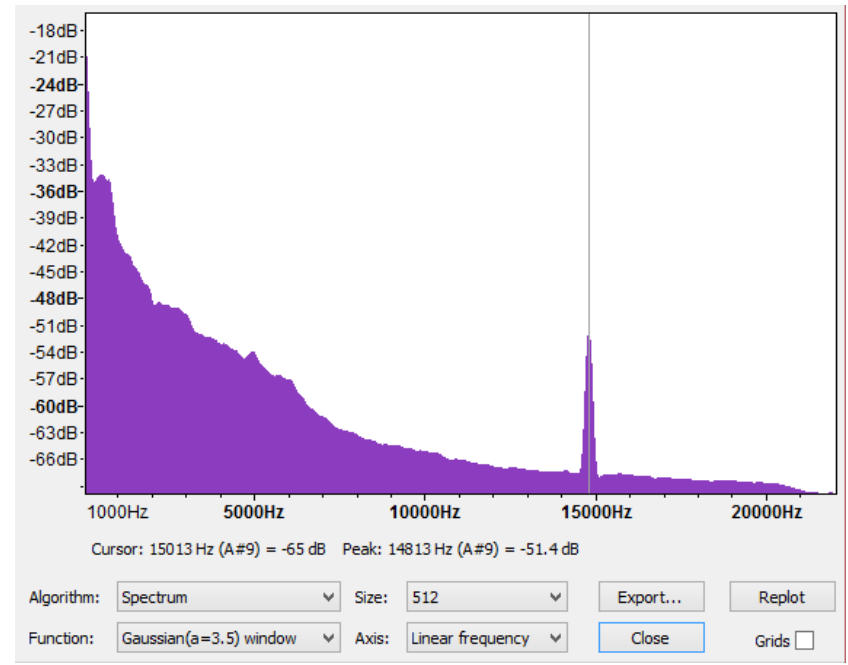

Figure 3 Spectrum frequency with photoacoustic signals processed with Audacity

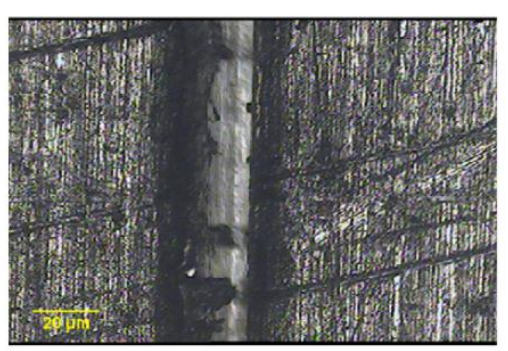

(a)

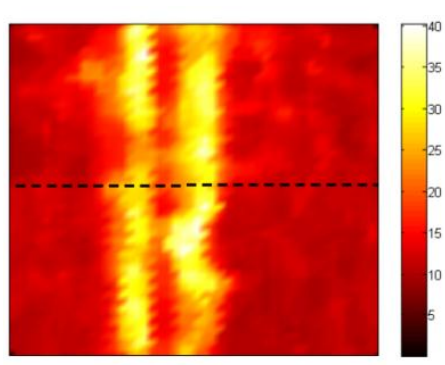

(b)

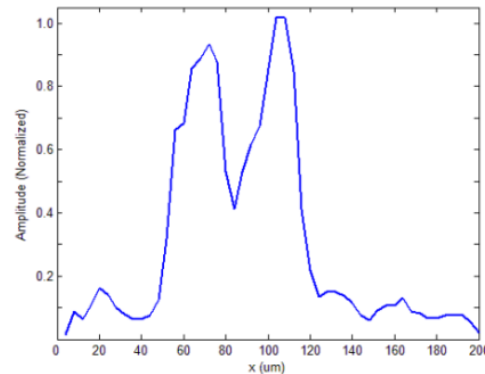

(c)

Figure 4 Visual imaging (a) shows a higher level of absorption at the edges of the cracks (more intense image). Acoustic imaging (b) shows stronger acoustic amplitude due to an increase in the deposited energy in the same area. Line graph (c) shows a cross-sectional image of the acoustic imaging (dashed 
After peak measurement, the object is set for 2D scanning to create an image of the crack. Figure 4a shows the visual image of a crack obtained with a microscope. The effect of greater absorption produced a darker image at the edges of the crack because the microscope uses optical observation, for which higher absorption causes a decrease in intensity level. This process is similar to photoacoustic imaging (Figure 4b), although the result is the opposite: the acoustic amplitude is higher at the edges of the crack than at the smooth surface in the middle.

These results align with the hypothesis that higher energy deposit due to photothermal activity produces stronger acoustic amplitude as a result of initial acoustic pressure. In these image analyses, the normal region shows an average amplitude of $-65 \mathrm{~dB}$, while at the edges of the crack it rises to $-40 \mathrm{~dB}$. This significant difference allows photoacoustic imaging to show cracks in a region due to high contrast. The cross-sectional image shown in Figure 4c shows the size of the crack. The curve shows a full width at half maximum (FWHM) of $60 \mu \mathrm{m}$. The measurement results are different from the actual conditions, likely because the laser beam is too wide. Consequently, the observation point is wider than planned, causing surface interaction with the area outside the observation object. The generated signal does not represent the point observed at the center of the laser. To solve this problem, the mechanical design of the collimator and $x-y$ stage need to be improved.

\section{CONCLUSION}

It is shown that the photoacoustic system designed in this experiment is able to create an image of a micro-crack on the surface of a material. Using simple devices, a laser diode and a commercial microphone can generate strong photoacoustic signals for data recording. Software has the potential to replace a lock-in amplifier for identifying signal peaks, making the system simpler and cheaper. The experiment resulted in a photoacoustic image with high contrast, clearly showing the crack. It is concluded that the surface of the material has a dominant effect on lasermaterial interaction compared to electronic factors. Thus, it is possible to use a photoacoustic system to learn about the surface of a material.

\section{ACKNOWLEDGMENT}

This work was supported by Research Program of the Ministry of Research, Technology and Higher Education, Republic of Indonesia.

\section{REFERENCES}

Beard, P., 2011. Biomedical Photoacoustic Imaging. Interface Focus, Volume 1, pp. 602-631

Bento, A.C., Dias, D.T., Olenka, L., Medina, A.N., Baesso, M.L., 2002. On the Application of the Photoacoustic Methods for the Determination of Thermo-optical Properties of Polymers. Brazilian Journal of Physics, Volume 32, pp. 783-802

Bergstrom, D., 2008. The Absorption of Laser Light by Rough Metal Surfaces. Doctoral Thesis. Department of Applied Physics and Mechanical Engineering, Luleå University of Technology, Luleå

Bouayoune, K.S., Boudi, E.M., Bachir, A., 2017. A Stochastic Method based on the Markov Model of Unit Jump for Analyzing Crack Jump in a Material. International Journal of Technology, Volume 8(4), pp. 622-633

Chigarev N., Zakrzewski, J., Tournat V., Gusev, V., 2009. Nonlinear Frequency-mixing Photoacoustic Imaging of a Crack. Journal of Applied Physics, Volume 12, pp. 254-268

Craig, B.D., Lane, R.A., Rose, D.H., 2006. Corrosion Prevention and Control: A Program Management Guide for Selecting Materials, Advanced Materials, Manufacturing, and Testing Information Analysis Center (AMMTIAC). Alion Science \& Technology, New York 
Favro, L.D., Kuo, P.K., Pouch, J.J., Thomas, R.L., Melander, N., Henningsen, J., 1980. A Photoacoustic Study of Absorption. Applied Physics Letters, Volume 36(12), pp. 45-60

Ganguly, P., Rao, C.N.R., 1981. Photoacoustic Spectroscopy of Solids and Surface. Journal of Chemical Sciences, Volume 90(3), pp. 153-214

Gdoutos, E.E., 2005. Solid Mechanics and Its Applications. Second Edition, Springer, Netherlands

Jeong, W.Y., Earls, C.J., Philpot, W.D., Zehnder, A.T., 2012. Inverse Thermographic Characterization of Optically Unresolvable Through Cracks in Thin Metal Plates. Mechanical Systems and Signal Processing, Volume 27, pp. 634-650

Kruusing, A., 2008. Handbook of Liquids-assisted Laser Processing. Elsevier, United Kingdom

Lantz, G., 2011. Crack Detection using a Passive Wireless Strain Sensor. Graduate Thesis. School of Civil and Environmental Engineering. Georgia Institute of Technology, Atlanta, Georgia, USA

LeBoulluec, P., Liu, H., Yuana, B., 2013. A Cost-Efficient Frequency-domain Photoacoustic Imaging System. American Journal of Physics, Volume 81(9), pp. 712-728

Lopez, J.A.B., Mandelis, A., Garcia, J.A., 2002. Normalized Photoacoustic Techniques for Thermal Diffusivity Measurements of Buried Layers in Multilayered Systems. Journal of Applied Physics, Volume 92(6), pp. 3047-3055

Luscher, E., Korpiun, P., Coufal, H.J., Tilgner, R., 1984. Photoacoustic Effect Principles and Applications. Springer Fachmedie. Wiesbaden, Germany

Maslov, K., Wang, L.V., 2008. Photoacoustic Imaging of Biological Tissue with IntensityModulated Continuous-wave Laser. Journal of Biomedical Optics, Volume 13(2), pp. 637644

Mezil, S., Chigarev, N., Tournat, V., Gusev, V., 2013. Two Dimensional Nonlinear Frequencymixing Photoacoustic Imaging of a Crack and Observation of Crack Phantoms. Journal of Applied Physics, Volume 114, pp. 1-17

Montalti, M., Credi, A., Prodi, L., Gandolfi, M.T., 2006. Handbook of Photochemistry. Third Edition, CRC Press, Boca Raton, FL, USA

Ni, G., 2014. Photoacoustic Measurement of Bandgaps of Thermoelectric Materials. Graduate Thesis. Department of Mechanical Engineering, Massachusetts Institute of Technology, Cambridge, Massachusetts, USA

Ristau, D., 2015. Laser-induced Damage in Optical Materials. CRC Press, Boca Raton, FL, USA Rupil, J., Roux, S., Hild, F., Vincent, L., 2011. Fatigue Microcrack Detection with Digital Image Correlation. Journal of Strain Analysis for Engineering Design, Volume 6(46), pp. 492-509

Setiawan, A., Suparta, G.B., Mitrayana, M., Nugroho, W., 2017. Subsurface Corrosion Imaging System based on Laser Generated Acoustic (LGA). International Journal on Advanced Science, Engineering and Information Technology, Volume 7(6), pp. 2189-2196

Sih, G.C., Chen, E.P., 1981. Mechanics of Fracture - Cracks in Composite Materials. Volume 6, Martinus Nijhoff Publishers, Netherlands

Tam, A.C., 1986. Application of Photoacoustic Sensing Techniques. Reviews of Modern Physics, Volume 58(2), pp. 381-431

Tolev, J., Mandelis, A., 2010. Laser Photothermal Non-destructive Inspection Method for Hairline Crack Detection in Unsintered Automotive Parts: A Statistical Approach. NDT\&E International, Volume 43, pp. 283-296

Vandenbussche, J.J, Lee, P., Peuteman, J., 2014. On the Accuracy of Digital Phase Sensitive Detectors Implemented in FPGA Technology. IEEE Transactions on Instrumentation and Measurement, Volume 63(8), pp. 1926-1936

Vocadlo, L., Poirer, J.P., Price, G.D., 2000. Grüneisen Parameters and Isothermal Equations of State. American Mineralogist Journal, Volume 85, pp. 1-6

Wang, L.V., 2009. Photoacoustic Imaging and Spectroscopy. CRC Press, Boca Raton, FL, USA 
Wang, T., Nandy, S., Salehi, H.S., Kumavor, P.D., Zhu, Q., 2014. A Low-cost Photoacoustic Microscopy System with a Laser Diode Excitation. Biomedical Optics Express, Volume 5(9), pp. 453-460

Yan, L., Gao, C., Zhao, B., Xingchen, M., Zhuang, N., Duan, H., 2012. Non-destructive Imaging of Standard Cracks of Railway by Photoacoustic Piezoelectric Technology. International Journal of Thermophysics, Volume 33, pp. 2001-2005 